\title{
Humeral Head Morphology Influences Outcomes of Arthroscopic Interposition Glenoid Patch Allograft for Glenohumeral Arthritis
}

\author{
Brian M. Cable, M.D., Ali S. Farooqi, B.A., Steven Tsai, B.S.E., Ryan Plyler, M.D., \\ Alex Lee, B.S., Robert L. Parisien, M.D., and John D. Kelly IV, M.D.
}

\begin{abstract}
Purpose: To determine short- to midterm patient-reported outcomes of arthroscopic soft-tissue interposition arthroplasty using acellular dermal allograft with a minimum follow-up of 1 year and to assess outcomes in patients with and without flattening of the humeral head. Methods: Patients with a diagnosis of primary glenohumeral arthritis who underwent arthroscopic soft-tissue interposition arthroplasty with an acellular dermal allograft from July 2010 to November 2019 were retrospectively enrolled. Inclusion criteria were a primary diagnosis of glenohumeral arthritis and Outerbridge 4 full-thickness cartilage loss of $\geq 50 \%$ of the glenoid articular surface. Patients underwent arthroscopic debridement, microfracture, and biological arthroscopic soft-tissue interposition arthroplasty with an acellular dermal matrix. Postoperative outcomes included American Shoulder and Elbow Surgeon (ASES) score, Single Assessment Numeric Evaluation (SANE) score, Penn Shoulder Score (PSS), numeric rating scale (NRS) pain score, analgesic use, and conversion to total shoulder arthroplasty (TSA). Results were stratified according to humeral head morphology on preoperative radiographs. Results: A total of 25 patients were included, with a mean age of 56.0 years (range 19.2 to 74.8) and a mean follow-up of 3.36 years (range 1.03 to 8.98). The mean postoperative ASES score was 64.1 (range 11.7 to 100.0 ), SANE score was $62 \%$ (range $5 \%$ to $100 \%$ ), and PSS was 61.2 (range 10.6 to 97.9 ). Additionally, $56 \%$ of patients rated their shoulder function as improved or much improved, and $36 \%$ of patients converted to TSA at a mean of 2.35 years. Patients with and without humeral flattening had similar postoperative ASES scores $(P=.44)$, SANE scores $(P=.90)$, PSS $(P=.73)$, and conversions to TSA $(P=.83)$. Patients with humeral flattening were more likely to have shoulder pain at night $(83.3 \%$ versus $28.6 \%, P=.02)$. Conclusion: Arthroscopic soft-tissue interposition arthroplasty with an acellular dermal allograft resulted in satisfactory short- to mid-term postoperative outcomes for younger patients with glenohumeral arthritis but demonstrated a TSA conversion rate of $36 \%$. Patients with humeral head flattening also had satisfactory shoulder function but were more likely to experience shoulder pain at night. Level of Evidence: Level IV, therapeutic case series
\end{abstract}

$\mathbf{O}$ steoarthritis $(\mathrm{OA})$ is a degenerative joint disease affecting joint cartilage, subchondral and periarticular bone, and periarticular soft tissues including ligaments, muscles, and synovium. ${ }^{1}$ OA of the glenohumeral joint (GHOA) is a frequent cause of shoulder pain resulting in severe functional impairment. Using the Samilson-Prieto radiographic criteria for the diagnosis of GHOA, recent epidemiologic studies suggested

From the Department of Orthopaedic Surgery, University of Pennsylvania, Philadelphia, Pennsylvania, U.S.A.

The authors report the following potential conflicts of interest or sources of funding: R.L.P. reports editorial or governing board, Arthroscopy, Arthroscopy, Sports Medicine, and Rehabilitation; board or committee member, American Orthopaedic Society for Sports Medicine, Society of Military Orthopaedic Surgeons; grantleducation, Arthrex. Full ICMJE author disclosure forms are available for this article online, as supplementary material. that GHOA may affect $\leq 16 \%$ to $20 \%$ of adults $>65$ years old. $^{2-4}$

Conservative measures for GHOA include corticosteroid injections, nonsteroidal anti-inflammatory drugs (NSAIDs), and physical therapy. Surgery is indicated after failure of conservative treatment, with anatomic total shoulder arthroplasty (TSA) or reverse total shoulder arthroplasty (RSA) being the mainstay of

Received January 8, 2021; accepted July 11, 2021.

Address correspondence to Robert L. Parisien, M.D., University of Pennsylvania, Philadelphia, PA 19103,U.S.A.E-mail: robert.l.parisien@gmail.com

(C) 2021 THE AUTHORS. Published by Elsevier Inc. on behalf of the Arthroscopy Association of North America. This is an open access article under the CC BY-NC-ND license (http://creativecommons.org/licenses/by-nc-nd/4.0/).

2666-061X/2118

https://doi.org/10.1016/j.asmr.2021.07.004 
surgical treatment for older, lower-activity patients. Studies have reported significant improvements in function and pain scores after TSA, with several studies reporting similar outcomes for patients after RSA and lower chances of revision surgery. ${ }^{5-8}$ Rates of surgery for GHOA have also dramatically increased, with some studies noting a $400 \%$ increase of primary TSA from 2001 to $2010 .^{9}$ In $2011,29,359$ TSA procedures and 21,692 RSA procedures were performed in the United States. ${ }^{10}$

Total arthroplasty is less reliable in younger patients $(<60$ years $)$ because of substantial activity restrictions and a low rate of survivorship, typically from loosening and wear of the glenoid component. ${ }^{11}$ Some studies have reported the 10-year survivorship of the glenoid component to be as low as $62.5 \%$ after TSA in young patients. ${ }^{12}$ Concerns regarding prosthetic survivorship and glenoid loosening have led surgeons to pursue joint-preserving procedures for younger patients instead. $^{13}$ One possible option is glenohumeral debridement, which allows for direct assessment of the joint surface and removal of damaged cartilage, osteophytes, and loose bodies, but $\leq 22 \%$ of patients convert to arthroplasty within a year of surgery. ${ }^{14}$ The comprehensive arthroscopic management procedure, which includes capsular release, biceps tenodesis, and axillary neurolysis in addition to glenohumeral debridement, is another alternative procedure that showed a lower conversion to arthroplasty rate of $15 \%$ at 2 years. ${ }^{15}$

Arthroscopic soft-tissue interposition arthroplasty, also known as biological glenoid resurfacing, is an additional option for the young patient with glenohumeral arthritis in which a majority of the wear is on the glenoid side. Allograft resurfacing of the glenoid is intended to create a biologically active articular surface that preserves motion while providing pain relief and improved function. ${ }^{16}$ Results of arthroscopic interposition arthroplasty have been mixed, ${ }^{17-20}$ although some studies have reported promising results with menis$\mathrm{cal}^{21-24}$ and dermal ${ }^{16,25}$ allografts. However, it is still unclear whether arthroscopic interposition arthroplasty is effective for patients with flattening of the humeral head, which has been associated with worse outcomes after other joint-preserving procedures. ${ }^{26}$

The purposes of this study were to determine short- to midterm patient-reported outcomes of arthroscopic soft-tissue interposition arthroplasty using acellular dermal allograft with a minimum follow-up of 1 year and to assess outcomes in patients with and without flattening of the humeral head.

\section{Methods}

Patients who underwent arthroscopic soft-tissue interposition arthroplasty with an acellular dermal allograft from July 2010 to November 2019, with a minimum follow-up of 1 year, were retrospectively enrolled in this institutional review board-approved study. Inclusion criteria were a primary diagnosis of glenohumeral arthritis and demonstrated Outerbridge 4 full-thickness cartilage loss $\geq 50 \%$ of the glenoid articular surface. Exclusion criteria were history of rheumatoid arthritis, history of psoriatic arthritis, reinjury to the involved shoulder, or injection of steroid or hyaluronic acid to the involved shoulder after initial surgery.

Postoperative outcome measures were collected retrospectively by telephone survey by a trained medical student. Recorded outcomes included postoperative American Shoulder and Elbow Surgeon (ASES) score, ${ }^{27}$ Single Assessment Numeric Evaluation (SANE) score, ${ }^{28}$ and Penn Shoulder Score (PSS). ${ }^{29}$ Patients rated their shoulder function and numeric rating scale (NRS) before and after surgery. Patients reported use of overthe-counter (OTC) analgesics and prescription pain medication before and after surgery. Nighttime shoulder pain was recorded. Subsequent surgery to the involved shoulder was also recorded during the survey, and the electronic medical record was used to obtain specific information about subsequent surgery. Patients with secondary surgery were asked to estimate their shoulder function and pain before the subsequent surgery. The number of patients meeting the patient acceptable symptom state (PASS), defined as the absolute postoperative outcome score that represents an acceptable clinical outcome, was reported using previously established thresholds for relevant patientreported outcomes. ${ }^{30}$

Preoperative radiographs, when available, were used to categorize patients based on humeral morphology. Two senior orthopaedic surgeons reviewed the preoperative radiographs and graded humeral morphology into two categories: zero to minimal humeral head flattening/osteophytes or significant humeral head flattening/osteophytes. Postoperative outcomes were then stratified by the presence or absence of significant humeral head flattening (Fig 1). Unpaired $t$ test for quantitative variables and chi-squared test for categorical variables were used for statistical analysis. All statistical analysis was completed using Prism 6.0 (GraphPad, San Diego, CA).

\section{Surgical Technique}

The surgical technique is illustrated in Fig 2. Patients underwent arthroscopic debridement, microfracture, and biological arthroscopic soft-tissue interposition arthroplasty with an acellular dermal matrix (Allopatch HD-ConMed). Patients were placed in the lateral decubitus position, and shoulders were prepped and draped in the usual fashion. Four portals were routinely made, including a standard posterior portal, a posterolateral portal, an anterior portal, and an anterior 
Fig 1. Radiographic comparison of humeral head flattening among
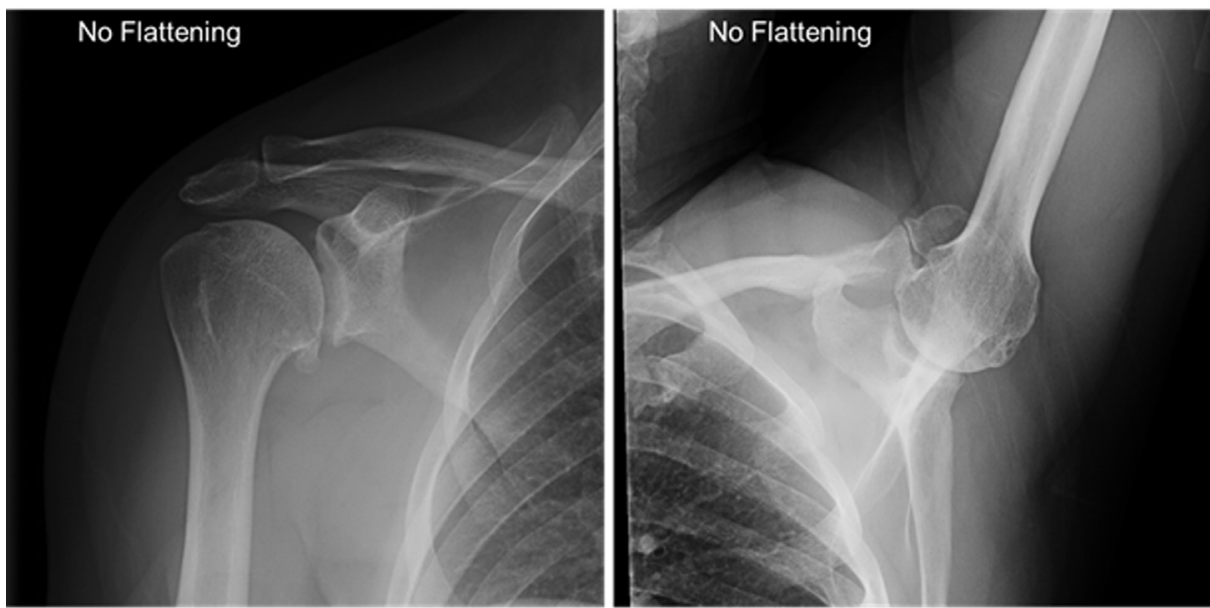
patients.
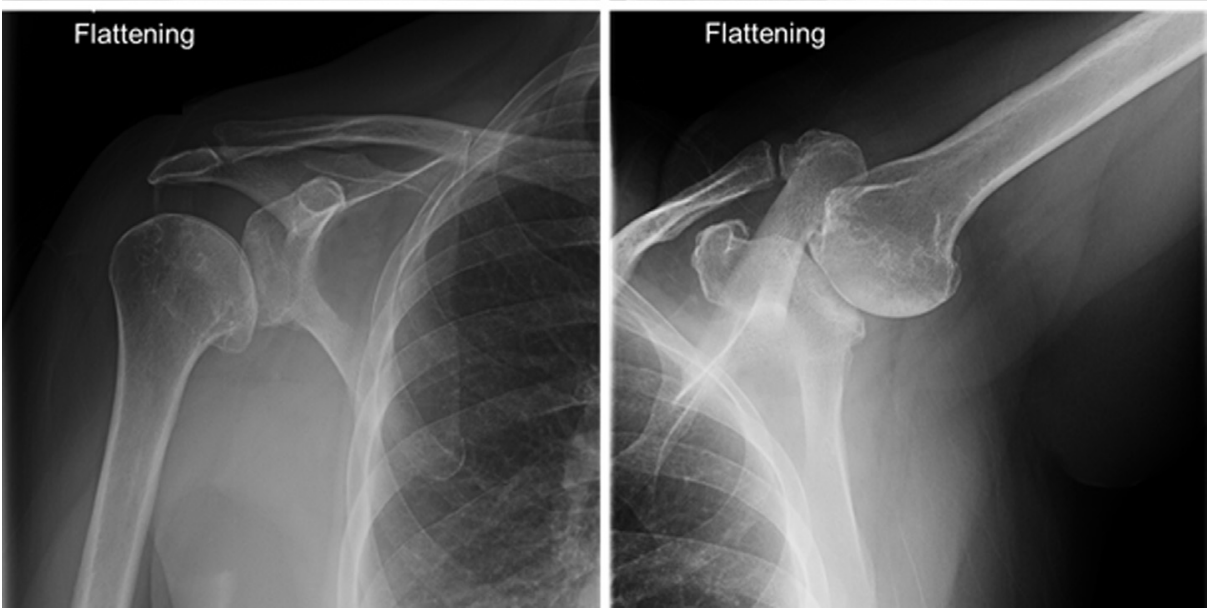

superior portal (ASP). Percutaneous portals were made as needed for anchor placement and suture retrieval. An extensive capsulotomy was performed with interval release to diminish joint reactive forces. Any evident humeral osteophytes were burred down via an accessory posterolateral portal while viewing posteriorly. Then the glenoid was carefully burred until concentric while viewing from the ASP. Usually, there was eccentric posterior wear and every attempt was made to make the glenoid more concentric and obtain bleeding bone. The labrum was debrided and usually maintained, and marrow stimulation via microfracture of the glenoid was performed. The $4 \times 3-\mathrm{cm}$ graft was then prepared on the back table, and 4 anchors were placed, the inferior ones percutaneously, with the superior anchors generally placed through either the posterior or anterior portal. A single limb of each inferior anchor was placed through the allograft, and a mulberry knot was tied so that the graft could be shuttled via the anterior cannula using a pulley technique. Once in the joint, sutures were shuttled through the proximal edges, and the graft was affixed to the superior labrum using push lock anchors (Arthrex, Naples, FL).
Additional sutures were applied securing the graft to the glenoid as needed.

\section{Rehabilitation}

Patients were placed into an abduction pillow, and gentle passive range of motion with applied manual traction was commenced at postoperative week 2 and continued until week 6 , at which point the sling was discarded. Active assisted range of motion began at week 6 , and resistive exercises were not instituted until week 12.

\section{Results}

In total, 34 patients underwent arthroscopic interposition arthroplasty during the study period, and 9 were lost to follow-up. The remaining 25 patients included for analysis had a mean follow-up of 3.36 years (range 1.03 to 8.98 ). Patients had a mean age of 56.0 years (range 19.2 to 74.8 ) and a mean follow-up of 3.36 years (range 1.03 to 8.98 ). Patients were majority male $(68.0 \%)$ and Caucasian $(88.0 \%)$, and the left shoulder was the most commonly affected $(56.0 \%)$. Available preoperative radiographs demonstrated 7 patients 

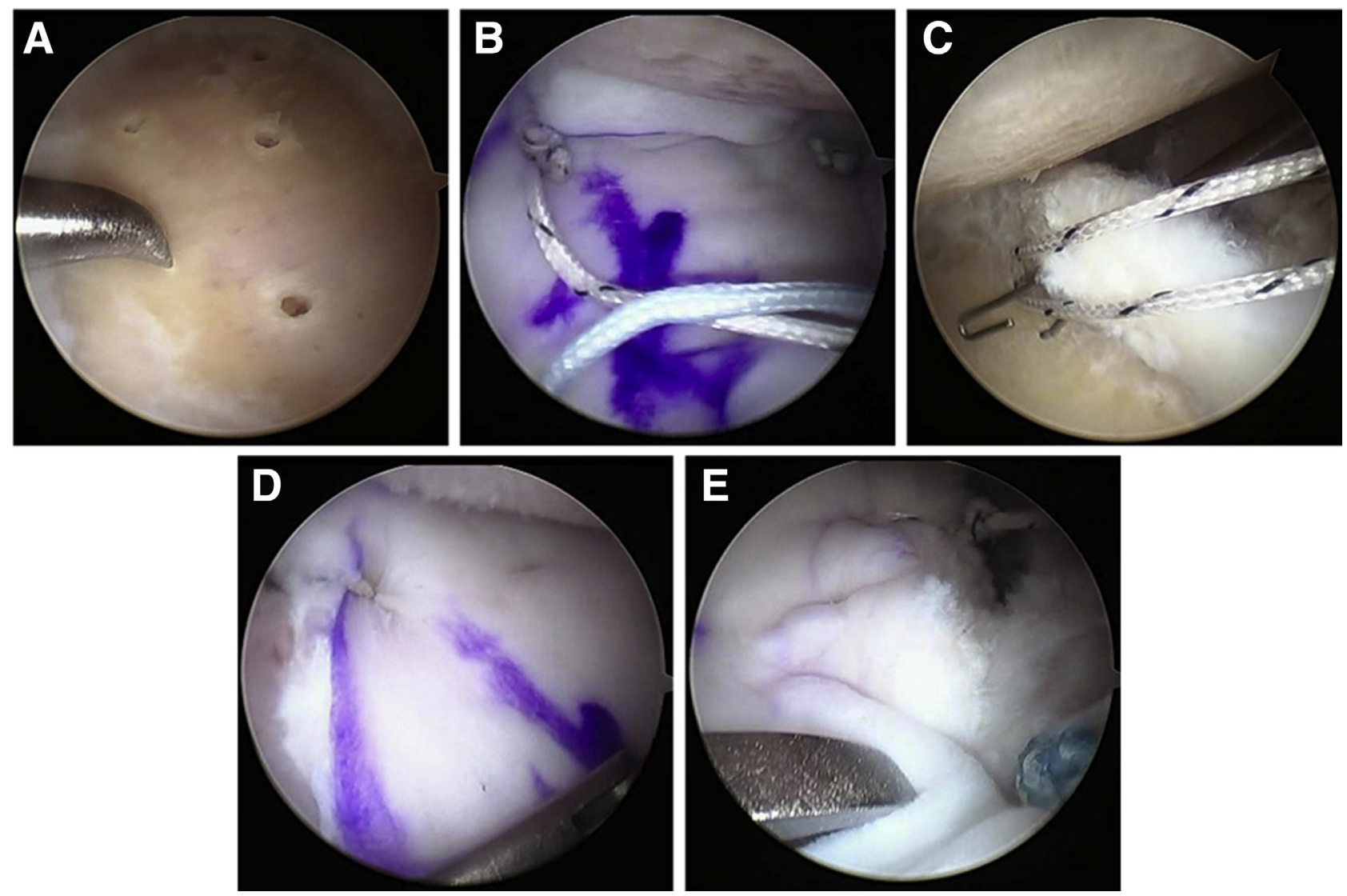

Fig 2. Arthroscopic images of surgical procedure demonstrating glenoid microfracture (A), graft shuttling using single limbs from the 2 inferior anchors tied as mulberry knots (B), and limb retrieval after graft shuttling (C). (D and E) Borders of the secured allograft.

without humeral head flattening and 12 patients with humeral head flattening. The mean ages of patients with and without flattening were 56.64 and 58.94 years, respectively $(P=.69) ; 66.7 \%$ and $57.1 \%$ were male $(P=.68)$; and $100 \%$ and $71.4 \%$ were Caucasian $(P=.15)$ (Table 1$)$.

The mean postoperative SANE score was $62 \%$ (range $5 \%$ to $100 \%$ ) for the involved shoulder and $90 \%$ (range $25 \%$ to $100 \%$ ) for the uninvolved shoulder. The PASS threshold for the SANE score was met in 7 of 25 patients. The mean postoperative ASES score was 64.1 (range 11.7 to 100.0), Eight of the 25 patients met the PASS threshold for the ASES score. The mean postoperative PSS score was 61.18 (range 10.57 to 97.89), with a mean score of 19.16 on the PSS pain subscale, 5.48 on the PSS satisfaction subscale, and 36.54 on the PSS function subscale (Table 2). Additionally, 56\% of patients reported their shoulder function as being improved or much improved, $20 \%$ reported shoulder function to be the same, and $24 \%$ reported shoulder function to be worse or much worse. When considering medication use, $40 \%$ of patients reported a decrease in OTC analgesic use, and $12 \%$ reported a decrease in prescription pain medication use. The mean reduction in pain NRS score was 3.44 , and $60 \%$ of patients reported shoulder pain at night. $36 \%$ of patients underwent conversion to TSA at a mean of 2.35 years (range 0.38 to 8.21 ); mean age at time of conversion was 57.28 years (range 37.94 to 74.83 ).

In a subanalysis of the 19 patients evaluated for humeral flattening on preoperative imaging, SANE scores were similar for the involved shoulder $67 \%$ and $66 \%$, $P=.90)$ and the uninvolved shoulder $(92 \%$ and $79 \%$, $P=.16)$. The PASS threshold for the SANE score was met by 3 of 12 and 2 of 7 patients with and without humeral flattening, respectively. Similarly, patients with and without flattening had a similar overall ASES (61.11 and 70.24, $P=.44$ ) and ASES function subscale (31.53 and 30.23, $P=.83$ ) scores. Patients with flattening had a slightly lower ASES pain subscale score (29.58) than patients without flattening (40.0), although this failed to reach significance $(P=.14)$. The PASS threshold for the ASES score was met by 2 of 12 and 3 of 7 patients with and without flattening, respectively. Patients with and without flattening demonstrated similar overall PSS (60.94 and 64.32, $P=.73$ ), PSS satisfaction subscale (5.75 and 6.14, $P=$ $.80)$, PSS function subscale (36.61 and 36.03, $P=.94$ ), 
Table 1. Demographics of patients undergoing interposition dermal allograft during arthroscopy, with statistical analysis comparing patients with and without humeral flattening

\begin{tabular}{|c|c|c|c|c|}
\hline Characteristic & Whole Population & Patients Without Humeral Flattening & Patients With Humeral Flattening & $P$ Value \\
\hline Patients & 25 & 7 & 12 & \\
\hline Age $(y)$ & $55.95(19.18$ to 74.83$)$ & $58.94(45.76$ to 73.88$)$ & $56.64(36.75$ to 74.60$)$ & .69 \\
\hline Duration of follow-up (y) & $3.36(1.03$ to 8.98$)$ & $2.13(1.16$ to 3.27$)$ & $2.60(1.03$ to 4.03$)$ & .36 \\
\hline Male & $17(68.0)$ & $4(57.1)$ & $8(66.7)$ & \\
\hline Female & $8(32.0)$ & $3(42.9)$ & $4(33.3)$ & \\
\hline Race & & & & .15 \\
\hline Asian & $1(4.0)$ & $1(14.3)$ & $0(0)$ & \\
\hline Affected shoulder & & & & .36 \\
\hline Right & $11(44.0)$ & $2(28.6)$ & $6(50.0)$ & \\
\hline Left & $14(56.0)$ & $5(71.4)$ & $6(50.0)$ & \\
\hline
\end{tabular}

Data are mean (range) or $\mathrm{n}(\%)$.

and PSS pain subscale $(18.58$ and $22.14, P=.24)$ scores (Table 1). After surgery, $66.7 \%$ of patients with flattening and $57.2 \%$ without flattening reported better shoulder function $(P=.39)$. Patients with flattening most commonly reported that their shoulder function was "improved" $(42.9 \%)$, whereas patients without

Table 2. Patient outcomes following glenoid resurfacing with interposition allograft. Statistical comparison was carried out for patients with and without humeral flattening.

\begin{tabular}{|c|c|c|c|c|}
\hline Outcome & Whole Population & Patients Without Humeral Flattening & $\begin{array}{c}\text { Patients With } \\
\text { Humeral Flattening }\end{array}$ & $P$ Value \\
\hline Conversion to arthroplasty & $9(36)$ & $2(28.6)$ & $4(33.3)$ & .83 \\
\hline Time to arthroplasty (y) & $2.35(0.38$ to 8.21$)$ & $1.26(0.76$ to 1.76$)$ & $2.26(1.05$ to 3.49$)$ & .37 \\
\hline $\begin{array}{l}\text { Age of patients undergoing } \\
\text { arthroplasty }(y)\end{array}$ & $57.28(37.94$ to 74.83$)$ & $58.06(52.75$ to 63.37$)$ & 53.55 (37.94 to 66.69$)$ & .66 \\
\hline \multicolumn{5}{|l|}{ SANE score } \\
\hline Involved shoulder & $62(5$ to 100$)$ & 66 (5 to 99$)$ & $67(30$ to 95$)$ & .90 \\
\hline Opposite shoulder & $90(25$ to 100$)$ & $79(25$ to 100$)$ & $92(70$ to 100$)$ & .16 \\
\hline PASS threshold & 7 of 25 & 2 of 7 & 3 of 12 & \\
\hline \multicolumn{5}{|l|}{ ASES score } \\
\hline Overall score & $64.13(11.67$ to 100.0$)$ & $70.24(13.33$ to 98.33$)$ & $61.11(31.67$ to 95.0$)$ & .44 \\
\hline Pain subscore & $32.20(0$ to 50.0$)$ & $40.0(10.0$ to 50.0$)$ & $29.58(10.0$ to 50.0$)$ & .14 \\
\hline Function subscore & $31.93(3.30$ to 50.0$)$ & $30.23(3.30$ to 48.30$)$ & $31.53(21.70$ to 45.0$)$ & .83 \\
\hline PASS threshold & 8 of 25 & 3 of 7 & 2 of 12 & \\
\hline \multicolumn{5}{|l|}{ Penn Shoulder Score } \\
\hline Total score & $61.18(10.57$ to 97.89$)$ & $64.32(34.0$ to 97.89$)$ & $60.94(27.0$ to 86.89$)$ & .73 \\
\hline Pain subscore & $19.16(2.0$ to 13.0$)$ & $22.14(12.0$ to 30.0$)$ & $18.58(10.0$ to 28.0$)$ & .24 \\
\hline Satisfaction subscore & $5.48(0$ to 10.0$)$ & $6.14(0$ to 10.0$)$ & $5.75(0$ to 10.0$)$ & .80 \\
\hline Function subscore & $36.54(7.0$ to 60.0$)$ & $36.03(7.0$ to 57.90$)$ & $36.61(10.0$ to 50.0$)$ & .94 \\
\hline Shoulder function rating & & & & .39 \\
\hline Much Improved & $7(28)$ & $3(42.9)$ & $2(16.7)$ & \\
\hline Improved & $7(28)$ & $1(14.3)$ & $6(50.0)$ & \\
\hline Same & $5(20)$ & $1(14.3)$ & $2(16.7)$ & \\
\hline Worse & $5(20)$ & $2(28.6)$ & $2(16.7)$ & \\
\hline Much Worse & $1(4)$ & $0(0)$ & $0(0)$ & \\
\hline Over-the-counter analgesic use & & & & .50 \\
\hline Fewer & $10(40)$ & $4(57.1)$ & $4(33.3)$ & \\
\hline Same & $14(56)$ & $3(42.9)$ & $7(58.3)$ & \\
\hline More & $1(4)$ & $0(0)$ & $1(8.3)$ & \\
\hline Prescription pain medication use & & & & .72 \\
\hline Fewer & $3(12)$ & $1(14.3)$ & $2(16.7)$ & \\
\hline Same & $20(80)$ & $6(85.7)$ & $9(75)$ & \\
\hline More & $2(8)$ & $0(0)$ & $1(8.3)$ & \\
\hline Reduction in NRS pain & $3.44(-2$ to 10$)$ & $5.43(-2$ to 10$)$ & 3.17 (2 to 7$)$ & .11 \\
\hline
\end{tabular}

Data are $\mathrm{n}(\%)$ or mean (range) unless noted otherwise.

ASES, American Shoulder and Elbow Surgeon; PASS, patient acceptable symptom state; NRS, numeric rating scale; SANE, Single Assessment Numeric Evaluation. 


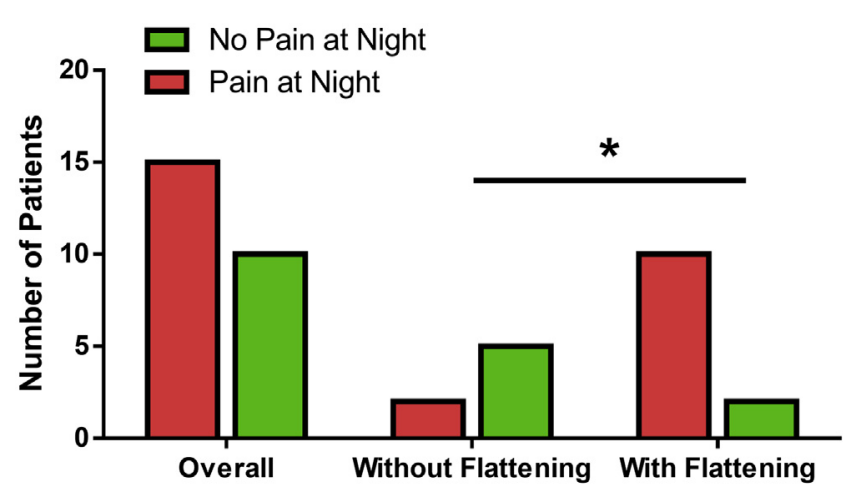

Fig 3. The number of patients experiencing shoulder pain at night after surgery. Patients with humeral flattening were significantly more likely to have shoulder pain at night compared with patients without humeral flattening $(P=.02)$.

flattening most commonly reported that their shoulder function was "much improved" (50.0\%).

Patients with flattening were less likely to report a decrease in OTC analgesic use $(33.3 \%)$ than patients without flattening $(57.1 \%)$, but this did not reach significance $(P=.50)$. Only a few patients in both cohorts reported a decrease in prescription pain medication use ( $16.7 \%$ and $14.3 \%, P=.72$ ). Patients with flattening reported a smaller mean reduction in the pain NRS subscale score (3.17) than patients without flattening (5.43) $(P=.11)$. However, a significantly greater number of patients with flattening experienced shoulder pain at night $(83.3 \%)$ than patients without flattening $(28.6 \%, P=.02)$ (Fig 3). Patients with and without flattening also converted to TSA at similar rates of $33.3 \%$ and $28.6 \%$, respectively $(P=.83)$. The mean time to TSA was 2.26 and 1.26 years for patients with and without flattening $(P=.37)$, respectively, and the mean age of patients at the time of TSA was 53.55 and 58.06 years $(P=.66)$.

\section{Discussion}

Arthroscopic soft-tissue interposition arthroplasty with an acellular dermal allograft resulted in acceptable short- to mid-term outcomes in younger patients with glenohumeral arthritis. However, $36 \%$ of patients converted to TSA at a mean of 2.35 years. Most patients reported an improvement in shoulder function, although many patients failed to reach the PASS threshold as evaluated by ASES or SANE score. Patients with humeral head flattening showed postoperative shoulder function comparable to that of patients without humeral head flattening as measured by patient-reported outcomes and conversion to TSA. However, patients with flattening were significantly more likely to experience shoulder pain at night.

Previous studies on outcomes after biological glenoid resurfacing vary by graft type and concomitant surgery, such as hemiarthroplasty, and have shown inconsistent results. Strauss et al. ${ }^{17}$ evaluated 41 patients after biologic glenoid resurfacing with meniscal or dermal allograft, with a mean follow-up of 2.8 years, and found significant postoperative improvements in VAS pain subscale score, ASES score, simple shoulder score (SST), forward elevation, and external rotation. However, they reported a clinical failure rate of $51.2 \%$ as defined by conversion to TSA, recommendation for TSA, revision surgery, persistence of disabling pain, or low postoperative ASES score. Longer-term studies of biological glenoid resurfacing have shown results similar to short-term studies. In an analysis of 30 patients after glenoid resurfacing with meniscal allograft at a mean follow-up of 8.3 years, Bois et al. ${ }^{31}$ reported significant improvements in shoulder function and reduction in pain, but also radiographic evidence of glenohumeral joint narrowing and a reoperation rate of $30 \%$.

Inconsistency results have been found after biologic glenoid resurfacing with concomitant hemiarthroplasty. Some studies have reported conversion to TSA rates as high as $60 \%$ to $83 \%$ after hemiarthroplasty and biologic glenoid resurfacing owing to persistent pain and loss of function resulting from glenohumeral joint space narrowing and glenoid erosion. ${ }^{32,33}$ Studies have also shown that patients undergoing biological glenoid resurfacing and concomitant hemiarthroplasty have worse clinical outcomes and failure rates than patients undergoing hemiarthroplasty alone. ${ }^{34}$ In a recent systematic review of 11 studies, Meaike et al. ${ }^{35}$ reported that glenoid resurfacing with concomitant hemiarthroplasty resulted in significant postoperative improvements of ASES score and visual analog scale (VAS) pain but was associated with an overall revision rate of $34 \%$ and rate of conversion to TSA of $22.7 \%$ at a mean time of 2.5 years. As demonstrated in our study, hemiarthroplasty may not be required to achieve satisfactory clinical outcomes in biological glenoid resurfacing procedures and may actually lead to worse outcomes, as reported in other studies.

Most recently, Hartzler et al. ${ }^{16}$ evaluated 43 patients undergoing glenoid resurfacing with human acellular dermal allograft with a mean follow-up of 5 years and found significant postoperative improvements in VAS pain score, ASES score, forward elevation, and external rotation and a conversion to TSA rate of $23 \%$. The postoperative VAS pain and ASES scores reported in this study are consistent with those of Hartzler et al., ${ }^{16}$ despite a slightly higher TSA conversion rate of $36 \%$. The high rates of conversion to TSA reported for this procedure are an important aspect to consider, because the subsequent TSA will be more technically challenging. This is especially true with regard to the present study, which found that the majority of patients converting to TSA were $<60$ years old. 
Previous studies have typically not evaluated the impact of humeral morphology on patient outcomes following biological glenoid resurfacing, although some have evaluated glenoid morphology. In a study of 19 shoulders that underwent glenoid resurfacing with meniscal allograft and hemiarthroplasty at a mean follow-up of 4.3 years, Lee et al. ${ }^{36}$ showed that patients with concentric glenoid wear had lower SST scores and higher VAS pain scores, although the differences were not statistically significant. In this study, we found that biological glenoid resurfacing with dermal allograft can be suitable for patients with humeral flattening but may not be as effective in reducing shoulder pain experienced at night. The finding that biological glenoid resurfacing may be suitable for patients with humeral head flattening is important, as humeral flattening limits treatment options and has been associated with worse outcomes after some procedures, such as comprehensive arthroscopic management (CAM). ${ }^{26}$ Not only does flattening of the humerus suggest advanced disease, it is also likely that the loss of a concentric joint increases shear stress on the graft and lowers survivorship. ${ }^{37}$

Lastly, the senior author believes that attention to detail in creating a spherical glenoid surface is critical to success and that many failures are a result of inadequate glenoid preparation. The slightly higher rate of conversion to TSA in this study may also be the result of inadequate glenoid preparation, particularly during the earlier years of this study, when this procedure was first being performed. The importance of creating a spherical glenoid surface is further supported by studies showing an increased risk of failure after arthroplasty for patients with loss of glenoid sphericity. ${ }^{38,39}$ Matsen et al. ${ }^{40}$ demonstrated that a ream-and-run procedure, consisting of humeral hemiarthroplasty with concentric reaming of the glenoid, can improve humeral head centering and patient outcomes. It is possible that the soft-tissue interposition arthroplasty procedure can be improved with a similar arthroscopic ream-and-run technique, which involves extralabral capsular release, drilling the glenoid centerline, and concentric reaming to restore sphericity. ${ }^{41}$

\section{Limitations}

This study had several limitations that may limit the generalizability of findings. There were only 25 patients included for analysis and 19 patients with available preoperative radiographs, thus limiting the statistical power of this study. There was no preoperative measurement of clinical function, such as ASES score or SANE score. As a result, there could have been differences not found in this study in preoperative function between patients with and without humeral flattening. There could also have been potential selection bias in this study, as patients were not excluded based on previous shoulder surgery or history of infection, and most patients were male and Caucasian. The wide range of patient age in this study may have also affected the outcomes.

Another limitation is that 7 patients had a follow-up of $<2$ years. This may have led to an underestimation of TSA conversion rate, which occurred at a mean of 2.35 years in this study. In addition, patients were asked to recall their preoperative NRS pain score, OTC analgesic use, and prescription pain medication use at follow-up. Patients may not have been able to reliably remember these preoperative measurements, and patient answers may have been subject to recall bias. Patients with subsequent surgery were also subject to further bias, as they estimated their shoulder function prior to additional surgery. However, this bias is likely to affect patients with and without humeral flattening equally and is not expected to affect the internal validity of this study. Another limitation is that the mean follow-up was only 3.36 years.

\section{Conclusion}

Arthroscopic soft-tissue interposition arthroplasty with an acellular dermal allograft resulted in satisfactory short- to mid-term postoperative outcomes for younger patients with glenohumeral arthritis, but demonstrated a TSA conversion rate of $36 \%$. Patients with humeral head flattening also had satisfactory shoulder function but were more likely to experience shoulder pain at night.

\section{References}

1. Ibounig T, Simons T, Launonen A, Paavola M. Glenohumeral osteoarthritis: An overview of etiology and diagnostics. Scand J Surg 2020:1457496920935018.

2. Cho HJ, Morey V, Kang JY, Kim KW, Kim TK. Prevalence and risk factors of spine, shoulder, hand, hip, and knee osteoarthritis in community-dwelling Koreans older than age 65 years. Clin Orthop Relat Res 2015;473:3307-3314.

3. Kobayashi T, Takagishi K, Shitara H, et al. Prevalence of and risk factors for shoulder osteoarthritis in Japanese middle-aged and elderly populations. J Shoulder Elbow Surg 2014;23:613-619.

4. Oh JH, Chung SW, Oh $\mathrm{CH}$, et al. The prevalence of shoulder osteoarthritis in the elderly Korean population: Association with risk factors and function. J Shoulder Elbow Surg 2011;20:756-763.

5. Walch G, Young AA, Melis B, Gazielly D, Loew M, Boileau P. Results of a convex-back cemented keeled glenoid component in primary osteoarthritis: Multicenter study with a follow-up greater than 5 years. J Shoulder Elbow Surg 2011;20:385-394.

6. Kiet TK, Feeley BT, Naimark M, et al. Outcomes after shoulder replacement: Comparison between reverse and anatomic total shoulder arthroplasty. J Shoulder Elbow Surg 2015;24:179-185. 
7. Triplet JJ, Everding NG, Levy JC, et al. Anatomic and reverse total shoulder arthroplasty in patients older than 80 years. Orthopedics 2015;38:e904-e910.

8. Favard L, Katz D, Colmar M, Benkalfate T, Thomazeau H, Emily S. Total shoulder arthroplasty to Arthroplasty for glenohumeral arthropathies: Results and complications after a minimum follow-up of 8years according to the type of arthroplasty and etiology. Orthop Traumatol 2012;98:S41-S47.

9. Schwartz BE, Savin DD, Youderian AR, Mossad D, Goldberg BA. National trends and perioperative outcomes in primary and revision total shoulder arthroplasty. Int Orthop 2015;39:271-276.

10. Westermann RW, Pugely AJ, Martin CT, Gao Y, Wolf BR, Hettrich CM. Reverse shoulder arthroplasty in the United States: A comparison of national volume, patient demographics, complications, and surgical indications. Iowa Orthop J 2015;35:1-7.

11. Brolin TJ, Thakar OV, Abboud JA. Outcomes after shoulder replacement surgery in the young patient: How do they do and how long can we expect them to last? Clin Sports Med 2018;37:593-607.

12. Denard PJ, Raiss P, Sowa B, Walch G. Mid- to long-term follow-up of total shoulder arthroplasty using a keeled glenoid in young adults with primary glenohumeral arthritis. J Shoulder Elbow Surg 2013;22:894-900.

13. Ansok CB, Muh SJ. Optimal management of glenohumeral osteoarthritis. Orthop Res Rev 2018;10:9-18.

14. Van Thiel GS, Sheehan S, Frank RM, et al. Retrospective analysis of arthroscopic management of glenohumeral degenerative disease. Arthroscopy 2010;26:1451-1455.

15. Millett PJ, Horan MP, Pennock AT, Rios D. Comprehensive arthroscopic management (CAM) procedure: Clinical results of a joint-preserving arthroscopic treatment for young, active patients with advanced shoulder osteoarthritis. Arthroscopy 2013;29:440-448.

16. Hartzler RU, Melapi S, de Beer JF, Burkhart SS. Arthroscopic joint preservation in severe glenohumeral arthritis using interpositional human dermal allograft. Arthroscopy 2017;33:1920-1925.

17. Strauss EJ, Verma NN, Salata MJ, et al. The high failure rate of biologic resurfacing of the glenoid in young patients with glenohumeral arthritis. J Shoulder Elbow Surg 2014;23:409-419.

18. Muh SJ, Streit JJ, Shishani Y, Dubrow S, Nowinski RJ, Gobezie R. Biologic resurfacing of the glenoid with humeral head resurfacing for glenohumeral arthritis in the young patient. J Shoulder Elbow Surg 2014;23:e185-e190.

19. Karelse A, Spapens N, Van Tongel A, De Wilde L. Arthroscopic treatment of the young degenerative shoulder joint; is there a role for interpositioning arthroplasty? Acta Orthop Belgica 2016;82:339-345.

20. Johnson D, Humphrey S, Norris T. Glenoid resurfacing with use of a lateral meniscal allograft. Tech Orthop 2007;22.

21. Pennington WT, Bartz BA. Arthroscopic glenoid resurfacing with meniscal allograft: A minimally invasive alternative for treating glenohumeral arthritis. Arthroscopy 2005;21:1517-1520.

22. Gobezie R, Lenarz CJ, Wanner JP, Streit JJ. All-arthroscopic biologic total shoulder resurfacing. Arthroscopy 2011;27:1588-1593.
23. Merolla G, Bianchi P, Lollino N, Rossi R, Paladini P, Porcellini G. Clinical and radiographic mid-term outcomes after shoulder resurfacing in patients aged 50 years old or younger. Musculoskel Surg 2013;97:23-29 (suppl 1).

24. Nicholson GP, Goldstein JL, Romeo AA, et al. Lateral meniscus allograft biologic glenoid arthroplasty in total shoulder arthroplasty for young shoulders with degenerative joint disease. J Shoulder Elbow Surg 2007;16: S261-S266.

25. de Beer JF, Bhatia DN, van Rooyen KS, Du Toit DF. Arthroscopic debridement and biological resurfacing of the glenoid in glenohumeral arthritis. Knee Surg Sports Traumatol Arthrosc 2010;18:1767-1773.

26. Arner JW, Elrick BP, Nolte PC, Haber DB, Horan MP, Millett PJ. Survivorship and patient-reported outcomes after comprehensive arthroscopic management of glenohumeral osteoarthritis: Minimum 10-year follow-up. Am J Sports Med 2021;49:130-136.

27. Kirkley A, Griffin S, Dainty K. Scoring systems for the functional assessment of the shoulder. Arthroscopy 2003;19:1109-1120.

28. Thigpen CA, Shanley E, Momaya AM, et al. Validity and responsiveness of the single alpha-numeric evaluation for shoulder patients. Am J Sports Med 2018;46:3480-3485.

29. Leggin BG, Michener LA, Shaffer MA, Brenneman SK, Iannotti JP, Williams GR. The Penn Shoulder Score: Reliability and validity. J Orthop Sports Phys Ther 2006;36: 138-151.

30. Polce EM, Wolfson TS, Skallerud WK, et al. Establishing thresholds for achievement of clinically significant satisfaction at two years following shoulder arthroplasty: The patient acceptable symptomatic state. Semin Arthroplasty JSES 2021;31:159-170.

31. Bois AJ, Whitney IJ, Somerson JS, Wirth MA. Humeral head arthroplasty and meniscal allograft resurfacing of the glenoid: A concise follow-up of a previous report and survivorship analysis. J Bone Joint Surg Am 2015;97:1571-1577.

32. Puskas GJ, Meyer DC, Lebschi JA, Gerber C. Unacceptable failure of hemiarthroplasty combined with biological glenoid resurfacing in the treatment of glenohumeral arthritis in the young. J Shoulder Elbow Surg 2015;24: 1900- 1907.

33. Elhassan B, Ozbaydar M, Diller D, Higgins LD, Warner JJ. Soft-tissue resurfacing of the glenoid in the treatment of glenohumeral arthritis in active patients less than fifty years old. J Bone Joint Surg Am 2009;91:419-424.

34. Hammond LC, Lin EC, Harwood DP, et al. Clinical outcomes of hemiarthroplasty and biological resurfacing in patients aged younger than 50 years. J Shoulder Elbow Surg 2013;22:1345-1351.

35. Meaike JJ, Patterson DC, Anthony SG, Parsons BO, Cagle PJ. Soft tissue resurfacing for glenohumeral arthritis: A systematic review. Shoulder Elbow 2020;12: 3-11.

36. Lee BK, Vaishnav S, Rick Hatch GF 3rd, Itamura JM. Biologic resurfacing of the glenoid with meniscal allograft: Long-term results with minimum 2-year follow-up. J Shoulder Elbow Surg 2013;22:253-260.

37. Farron A, Reist A, Terrier A. Consequences of humeral head flattening due to osteoarthritis. Orthop Proc 2009;91B:160-160. 
38. Iannotti JP, Norris TR. Influence of preoperative factors on outcome of shoulder arthroplasty for glenohumeral osteoarthritis. J Bone Joint Surg Am 2003;85: 251-258.

39. Donohue KW, Ricchetti ET, Iannotti JP. Surgical management of the biconcave (B2) glenoid. Curr Rev Musculoskelet Med 2016;9:30-39.
40. Matsen FA 3rd, Warme WJ, Jackins SE. Can the ream and run procedure improve glenohumeral relationships and function for shoulders with the arthritic triad? Clin Orthop Relat Res 2015;473:2088-2096.

41. Matsen FA 3rd, Lippitt SB. Current technique for the ream-and-run arthroplasty for glenohumeral osteoarthritis. JBJS Essent Surg Tech 2012;2:e20. 